\title{
Analysis of Three-dimensional Doppler Ultrasonographic Quantitative Measures for the Discrimination of Prostate Cancer
}

\author{
Aaron P. Moskalik, PhD, Mark A. Rubin, MD, Kirk J. Wojno, MD, \\ Robert Bree, MD, Jonathan M. Rubin, MD, PhD, J. Brian Fowlkes, PhD, \\ James E. Montie, MD, Sargum Manley, MS, Paul L. Carson, PhD
}

\begin{abstract}
Abbreviations
$C P D_{\mathrm{fs}^{\prime}}$ color pixel density in frequency shift; $\mathrm{CPD}_{\mathrm{pm}^{\prime}}$ color pixel density in power mode; $\mathrm{H} \& \mathrm{E}$, hematoxylin-eosin NMPCP, normalized mean power in color pixels; ROC receiver operating characteristic; 3D, three-dimensional; $\checkmark$, mean velocity in color pixels

Received September 18, 2000, from the Departments of Basic Radiological Sciences (A.P.M., J.B.F., P.L.C.), Pathology (M.A.R., S.M.), Radiology (J.M.R.), and Urology (J.E.M.), University of Michigan, Ann Arbor, Michigan; Department of Radiology, University of Missouri Health Sciences Center, Columbia, Missouri (R.B.); and Department of Pathology, St John Hospital, Detroit, Michigan (K.J.W.). Revision requested December 7, 2000. Revised manuscript accepted for publication February 20, 2001.

This work was supported in part by the Specialized Program of Research Excellence in Prostate Cancer (US Public Health Service grant 1P50 CA69568) funded by the National Cancer Institute.

Address correspondence and reprint requests to Aaron Moskalik, PhD, Department of Basic Radiological Sciences, University of Michigan, 200 Zina Pitcher Pl, Kresge III, Room 3316, Ann Arbor, MI 48109-0553.
\end{abstract}

Objective. The purpose of this study was to determine whether several quantitative ultrasonographic measures have potential to discriminate prostate cancer from normal prostate and to determine the best combination of these measures. The true spatial distributions of cancer within the prostates studied were obtained histologically after radical prostatectomy. The relationship between Doppler ultrasonography and microvessel count was also investigated. Methods. Three-dimensional Doppler ultrasonographic data were acquired from 39 patients before radical prostatectomy. The removed prostate was sectioned, and whole-mount hematoxylineosin-stained slides were used to identify all regions of cancer within each prostate. These histologic and ultrasonographic data were spatially registered. Doppler ultrasonographic measures were calculated within uniformly sized three-dimensional regions that were either entirely cancerous or noncancerous, and receiver operating characteristic analysis was performed on the results. Microvessel counts were made within each contiguous cancerous region and correlated with ultrasonographic measures. Results. Color pixel density was the best simple measure for discriminating prostate cancer (accuracy, $80 \%$ ). The mean power mode value (normalized mean power in color pixels) was inversely related to cancer with an accuracy of 1 - normalized mean power in color pixels $=65 \%$ (low mean power is more cancerous). When color pixel density was combined with the normalized mean power in color pixels, its accuracy improved slightly to $84 \%$. The peak microvessel count had a negative correlation with color pixel density as well as with cancer stage. Conclusion. Doppler ultrasonography does provide discriminatory information for prostate cancer, with color pixel density being the most promising measure. Key words: prostate, ultrasonography; prostate, neoplasms; ultrasonography, three-dimensional; ultrasonography, Doppler studies; ultrasonography, comparative studies; ultrasonography, power Doppler studies. 
$\mathrm{P}$ rostate cancer is the most frequently diagnosed cancer in men. In North America it has the highest mortality rate of all cancers in men except for lung cancer. ${ }^{1}$ When prostate cancer is diagnosed early, it is usually curable. Treatment is often effective even at later stages. Therefore, the decision of who and when to treat for prostate cancer is very important.

These decisions are not made easily for 2 reasons. First, prostate cancer is highly prevalent. As much as $30 \%$ of the male population older than 50 years have prostate cancer found at autopsy. ${ }^{2}$ Second, prostate cancer progresses relatively slowly. Of the $30 \%$ with prostate cancer found at autopsy, most died of other causes, and the cancer detected was typically of a volume of less than $0.5 \mathrm{~cm}^{3}$. Therefore, detection of prostate cancer alone does not mean the best course of action is treatment. The stage and aggressiveness of the cancer also must be taken into account.

The current methods of prostate cancer diagnosis do not adequately provide the information needed to make informed treatment decisions. Diagnostic techniques include transrectal ultrasonography, prostate-specific antigen measurement, and transrectal needle biopsy. Currently, B-mode ultrasonography is used to look for hypoechoic regions of the prostate. However, not all cancer appears hypoechoic, especially the smaller masses. ${ }^{3}$ Prostate-specific antigen production can also be influenced by factors such as acute prostatitis, acute urinary retention, rigid cytoscopy, transrectal ultrasonography, transrectal needle biopsy, surgery, and ejaculation. ${ }^{2} \mathrm{~A}$ biopsy core cannot provide information about the extent or prevalence of the cancer. Because of this, there is some debate as to how many biopsies are adequate for a given patient. ${ }^{4}$

Doppler ultrasonography, both power mode and frequency shift, has also been studied for diagnosis of prostate cancer. The rationale for using color flow imaging is that cancer will develop an increased blood supply by angiogenesis, and this can show up as increased and abnormal color flow patterns in and around the cancerous regions.

In the prostate, color flow imaging has been tried with simple visual staging schemes. This technique has had some success in discriminating cancer from benign tissue ${ }^{5}$ and in determining cancer prognosis. ${ }^{6,7}$ More recently a study was done in which hypervascular lesions as seen on power Doppler imaging correlated with increases in biopsy yields. ${ }^{8}$
This study was designed to evaluate several different components of Doppler ultrasonographic information as discriminators for prostate cancer. The purposes behind the results of the study, therefore, were to provide some insight into what information provided by Doppler imaging may be useful for this task and to point the way toward an effective ultrasonographic diagnostic tool for prostate cancer. However, this study was not designed to determine the efficacy of Doppler ultrasonography in any specific clinical context.

Additionally, in this study we investigated the relationship between quantitative ultrasonographic measures and peak microvessel counts from the same cancerous region to determine whether the information seen on Doppler ultrasonography is related to the location of the smallest vessels as seen on histologic examination.

\section{Materials and Methods}

\section{Study Population}

Fifty-two patients who were to undergo radical prostatectomy were recruited for this study. For 12 patients, 1 or more of the three-dimensional (3D) data sets required for analysis were missing; consequently, these 12 patients were excluded. No cancer was found in another patient after prostatectomy. Four patients were treated hormonally before this study, but excluding them from the data set did not significantly alter the results.

All patients included in this study were referred to the local department of urology for radical prostatectomy as part of their treatment. This study was not racially screened; nevertheless, all subjects were white. Institutional Review Board approval was obtained, as was informed consent from all patients before ultrasonography.

\section{Acquisition}

The first step was to gather the raw volume data necessary to compute the various ultrasonic measures and to histologically identify cancerous regions. Data volumes acquired in this study included the in vivo frequency shift and power mode ultrasonographic scans, the images of the histologic hematoxylin-eosin (H \& E)-stained slides, the factor VIII-stained slides, and the in vitro ultrasonographic scans used to register the histologic images with the in vivo scans (Fig. 1). 


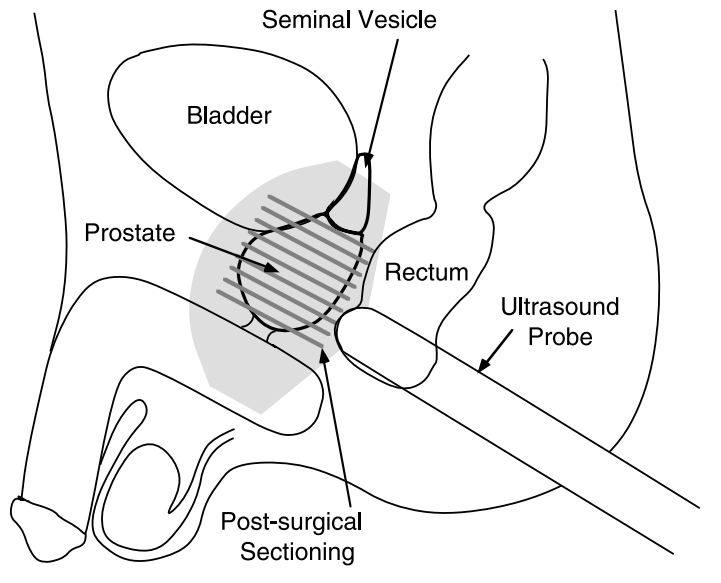

Figure 1. The prostate is shown in its anatomic position. The ultrasonic probe was inserted transrectally and produced images of the area shown in gray above. The probe was rotated at the anus from left to right, so that the entire prostate was imaged. After prostatectomy, the gland was ultrasonographically imaged and sectioned in the orientation shown by the gray lines.

The frequency shift scan was acquired with an ATL HDI 3000 system (ATL Ultrasound, Bothell, WA) using a C9-5 transrectal probe. In a 3D scan of the prostate, the transducer was rotated so that the scan plane swept through the entire prostate; the axis of rotation was positioned at the anus for minimum discomfort. The apparatus described by Moskalik et $\mathrm{al}^{9}$ was developed to acquire the desired in vivo image slices in a stable, reproducible way so that scan slices can later be reconstructed in the correct geometric configuration.

The physician conducting the scan manually controlled the angular position. A digital readout displayed the relative angular position of the probe from the beginning of the scan. At fixed increments of $0.54^{\circ}$, the color flow image at that position was saved as a TIFF file to the internal hard disk of the ATL system. The acquired image was selected manually at peak systole. For larger prostates, the increment was increased to $0.72^{\circ}$ to keep the scan time short and to reduce patient discomfort. The scan plane was oriented sagittally and was rotated in this manner from right to left through the prostate so that the entire gland was imaged.

The settings of the ATL system were kept consistent for all scans. On the basis of the experience of the radiologists involved in this study, the following settings were used. The general prostate preset was selected. The pulse repetition frequency was set to $1000 \mathrm{~Hz}$ for the frequency shift scan and $700 \mathrm{~Hz}$ for the power mode scan. The gain was occasionally adjusted to reduce noise, but typically it was set to $70 \%$ for frequency shift and $84 \%$ for power mode. The wall filter settings were low and medium for frequency shift and power mode, respectively. Persistence and frame rate were set at medium, and the sensitivity was set at high.

After the in vivo scans of the prostate, the ultrasonographic volume data existed as a series of TIFF images. The color flow information was encoded in these images as different red-green-blue values whose power or velocity value was assigned in a color bar along the side of each image. This color information was written over the gray scale B-mode information.

The power mode and frequency shift information encoded in the color part of the images was the raw form of the data that were to be correlated with the histologic data. The gray scale B-mode part of the images was needed for the spatial registration of the ultrasonographic images with the histologic slides.

The first processing step for the raw in vivo images was to separate the color information from the gray scale information and to decode the red-green-blue color information into the scalar intensity or speed by using the color bar. The next step was to reconstruct the images, which were acquired by an angular sweep, into a rectilinear field. Finally, color artifacts such as the reverberation found behind calcifications were removed from the color flow data. This was accomplished by using custom software written in the AVS/Express (Advanced Visual Systems, Waltham, MA) environment.

After the prostate was surgically removed and placed in a stabilizing holder for positioning and sectioning, it was rescanned in vitro by using the HDI 3000 system with the same C9-5 probe used for the in vivo scans. The prostate was scanned linearly with evenly spaced slice planes (0.5-mm spacing) in the same orientation and essentially the same planes as for the histologic sections. This made it easier to later align the histologic slides by using the in vitro scan.

The prostate was then sliced into $3-\mathrm{mm}$ sections in the same transverse orientation. From each of these sections, an $\mathrm{H} \& \mathrm{E}$-stained slide was made. On the H \& E-stained slide, the can- 
cerous lesions were outlined and graded by a urologic pathologist.

The left, right, and posterior aspects were also labeled. This information coupled with the in vitro ultrasonographic scan was used to align the images of the histologic slides into a 3D volume. Matching the position of the urethra on adjacent slides further refined the alignment of the slides.

The next step was to form a 3D field where the voxels within each cancerous volume were labeled. The cancer outline markings were selected by a filter, and the cancer volumes were filled in. Each individual cancer volume was then assigned a different label. Adjacent cancerous regions from different slides were given the same label. This provided a 3D mapping of where the cancer was located. The region extending $0.5 \mathrm{~cm}$ out from each cancerous region was also uniquely labeled in this map.

\section{Registration}

A manual registration technique was developed to spatially align the ultrasonographic and histologic data sets. ${ }^{10}$ This technique modeled the prostate with an ellipsoid that could be used to generate an affine transform matrix (Fig. 2).

The power mode data volume was used as the reference volume onto which the frequency shift and histologic data volume was registered. The transform for either of these 2 registrations was computed by multiplying the inverse transform of the power mode ellipsoid definition matrix by the ellipsoid definition matrix from the frequency shift or histologic volume.

A reconstructed volume was generated for the frequency shift and the histologic data, with the $3 \mathrm{D}$ field identical in size to the power mode field. The value of each voxel in a reconstructed field was computed by multiplying the vector position of that voxel by the transform matrix. This process can be represented by the following equation:

$$
\vec{v}_{r} E_{o} E_{r}^{-1}=\vec{v}_{o}
$$

where $\vec{v}_{r}$ was the vector in the reference space (power mode data set); $E_{o}$ was the ellipsoidal matrix of the original prostate volume; $E_{r}$ was the ellipsoid matrix of the reference prostate; and $\vec{v}_{o}$ was the corresponding position vector in the original volume.
The transform matrix was refined by directly comparing the ultrasonographic and histologic data within the same volume and visually assessing the quality of the registration. Adjustments were made to the transform matrix accordingly.

The histologically defined cancer mapping could then be mapped directly onto the ultrasonographic data, producing a spatial mapping that related the color flow data to the identified cancerous regions.

\section{Quantitative Ultrasonographic Measures}

The information used in the quantitative ultrasonographic measures can be classified as 2 types: the mean color flow value within a region and the color flow density within a region. This coupled with the 2 types of Doppler images acquired, frequency shift and power mode, produced 4 basic measures: mean velocity in color pixels $(\mathrm{V})$, normalized mean power in color pixels (NMPCP), color pixel density in frequency shift $\left(\mathrm{CPD}_{\mathrm{fs}}\right)$, and color pixel density in power mode $\left(\mathrm{CPD}_{\mathrm{pm}}\right)$. These measures were computed by the following equations:

$$
\begin{gathered}
C P D=N_{b} / N_{t} \\
V=\sum_{i=1}^{N_{b}} V_{i} / N_{b} \\
N M P C P=\sum_{i=1}^{N_{b}} P_{i} /\left(P_{b} \cdot N_{t}\right),
\end{gathered}
$$

where $N_{b}$ was the number of color pixels in the region of interest; $N_{t}$ was the total number of pixels in the region of interest; $V_{i}$ was the estimated mean velocity of moving particles in the ith pixel as represented by the color of that pixel; and $P_{i}$ was the linearized power value of the ith pixel. $P_{b}$ was a threshold value taken to represent the power signal from a region of $100 \%$ blood at a given depth. This value was used as a normalization factor to correct for depth dependencies such as attenuation in the power signal. The normalization factor was computed in 5 depth zones for each prostate by using the method described by Rubin et al. ${ }^{11}$

Briefly, this method used the cumulative distribution of power mode values found within a depth zone. The "knee" of this distribution could 
be found reproducibly as a measure of local signal level. $P_{b}$ was designed to correspond to the power value seen in the high sheer region of large vessels, where it could be reasonably assumed that any power greater than this knee value represented $100 \%$ blood; i.e., that the entire voxel was within a large blood vessel without rouleaux. Whether that interpretation was appropriate for the small vessels studied here has not been determined. $P_{b}$ appeared to function stably as an adjustment for signal level with depth. Any effect of the absolute value of $P_{b}$ as a function of depth was eliminated by the subsequent normalization described below.

For frequency shift measures, an isotropic blood flow direction was assumed within each Doppler sample volume. This assumption did not hold for the larger vessels; however, statistically over all prostate volumes, one would expect an isotropic distribution, and therefore, our analysis should not be affected by this assumption.

Average ultrasonographic color flow measures vary significantly among patients despite carefully maintaining consistent machine settings. This may be due to systemic differences among patients' circulation, the timing of the ultrasonographic scan relative to physiologic events such as ejaculation, and local differences in the circulation to the prostate due to cancer or other disease. Because these different factors were not and could not be controlled in this study, they could not be separated and accounted for individually. Therefore, all the quantitative measures for each prostate were normalized by the value calculated within the total noncancerous region of that prostate. All other regions' measures were expressed relative to the noncancerous tissue's mean value within that prostate.

Further compound measures can be computed by combining the above measures, for instance, speed-weighted pixel density was $\mathrm{V} \cdot \mathrm{CPD}_{\mathrm{fs}}$, and normalized power-weighted pixel density was NMPCP $\cdot \mathrm{CPD}_{\mathrm{pm}}$.

\section{Receiver Operating Characteristic Analysis}

The next step was to determine which of these quantitative measures provided information that can be used to discriminate cancer from noncancerous tissue in the prostate and which measure or combination of measures did this best. A powerful tool appropriate for this type of analysis is the receiver operating characteristic (ROC) curve. ${ }^{12}$

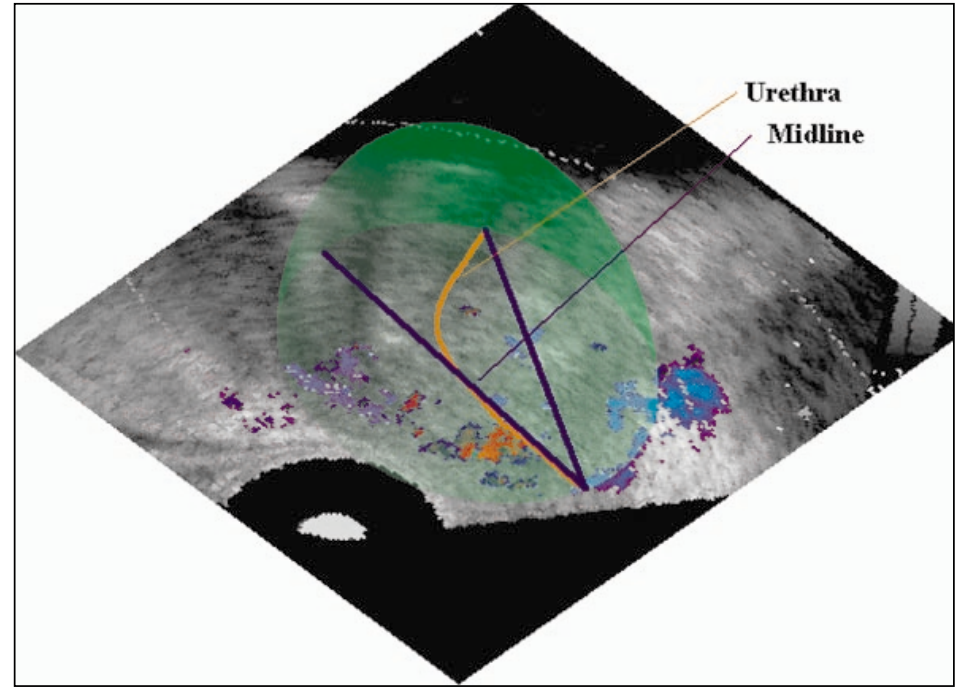

Figure 2. Representation of prostate model. A midsagittal slice of the reconstructed power mode data is shown, with the transparent green ellipsoid shell representing the prostate model. The beige line marks the location of the urethra. The left blue line was drawn at the midline of the ellipsoid. The right blue line connects the ends of the prostatic urethra. The ellipsoid is positioned so that the angle between these 2 lines is $30^{\circ}$. The dark B-mode line to the left and roughly parallel to the urethra is due to the boundary between the peripheral zone and the central zone, which can sometimes be easily confused with the urethra.

An ROC curve is essentially a plot of the truepositive fraction versus the false-positive fraction for a range of threshold values from the measure being investigated as a discriminator. An ideal detector would have an ROC curve with a true-positive fraction of 1 at a false-positive fraction of 0 . The area under this curve would be 1 . A detector that is unrelated (provides no discrimination) to the quality that is being discriminated would have an ROC curve that runs diagonally from $(0,0)$ to $(1,1)$ and an area of 0.5 under it. Real detectors have curves that fall between these 2 extremes, with better detectors having a larger area.

To apply this technique to our data, first we divided each prostate into equally sized volumes $\left(0.8 \mathrm{~cm}^{3}\right)$, which were either entirely cancerous or entirely noncancerous as defined by the histologically identified regions of cancer. ${ }^{13}$ These volumes were contiguous and as compact as possible. This was accomplished by using the mapping of prostate cancer identified on the histologic slides to define the boundaries between the cancer and the normal tissue. The $0.5-\mathrm{cm}$ shell around each cancerous region was excluded in this analysis. 
The various quantitative measures were computed for each volume from all the prostates. These data were then fed into the ROC analysis package ROCkit (Charles Metz, Department of Radiology, University of Chicago, Chicago, IL).

\section{Microvessel Density}

In our study, whole-mount factor VIII-stained slides were made of each prostate from the 3$\mathrm{mm}$ sections. A 0.5-cm grid was drawn onto each slide. By using a method similar to that used by Weidner et al, ${ }^{14}$ microvessel counts were made. Within each grid square that contained cancer, a region was selected at low microscopic power that contained the visually greatest amount of endothelial stain. This area was then examined at $\times 200$ magnification, and the number of distinguishable microvessels was counted. Likewise, a count was made in the area outside the cancer within this square. In addition, a count was made within 5 noncancerous squares on each slide. This resulted in a number of counts for each cancerous volume and the noncancerous portion of each prostate (1 count per $0.5-\mathrm{cm}$ square); subsequent discussion of the distribution of counts refers to the sets of counts associated with a cancerous or noncancerous region.

Microvessel density provides information about the distribution of the smallest blood vessels, where Doppler ultrasonography detects only larger vessels. The next question to be answered was whether the presence of the larger ultrasonographically visible blood vessels within a cancer correlated with high microvessel counts.

To answer this question, various ultrasonographic quantitative measures were computed within each cancerous region and correlated with the 90th-percentile microvessel count from that same region. The cancerous regions were separated by size; cancers with volumes greater than $0.5 \mathrm{~cm}^{3}$ were labeled large, and those with volumes less than $0.5 \mathrm{~cm}^{3}$ were labeled small.

Additionally, each prostate was classified as either adverse disease, meaning it was found to have cancer-positive margins, an extraprostatic extension, or cancer-positive seminal vesicles, or organ confined. The microvessel counts within each prostate were subdivided by tissue type: noncancerous, adjacent to cancer, Gleason grades 4 and 5, Gleason grade 6, and Gleason grades 7 to 9 . The fraction of prostates with each tissue type and a microvessel density greater than 80 is reported in "Results."

\section{Results}

The first results presented here were designed to show what aspects of the ultrasonographic color flow data provide information that will aid in the detection of prostate cancer. As described above, each prostate was divided into $0.8-\mathrm{cm}^{3}$ volumes that were either entirely cancerous or noncancerous. The 4 basic measures, V, NMPCP, $\mathrm{CPD}_{\mathrm{f}}$, and $\mathrm{CPD}_{\mathrm{pm}}$, were computed within each volume. From these data, an ROC analysis was performed (Fig. 3).

It can be readily seen that $\mathrm{CPD}_{\mathrm{fs}}$ was the best basic measure for discriminating prostate cancer, with an accuracy of $80 \%$. Both $\mathrm{CPD}_{\mathrm{pm}}$ and $\mathrm{V}$ provided very little discriminating value, with levels of accuracy of $58 \%$ and $54 \%$, respectively. NMPCP had an accuracy of $35 \%$, which implies that a low NMPCP correlates with cancer (i.e., 1 - NMPCP had an accuracy of $65 \%$ ).

The second results combined the 2 basic measures that showed promise, $\mathrm{CPD}_{\mathrm{fs}}$ and NMPCP, into a Bayesian linear discriminator of the form $\mathrm{CPD}_{\mathrm{fs}}-3.3 \cdot$ NMPCP. Figure 4 shows the ROC curve of this measure compared with those of $\mathrm{CPD}_{\mathrm{fs}}$ and 1/NMPCP. The accuracy of $\mathrm{CPD}_{\mathrm{fs}}-3.3 \cdot \mathrm{NMPCP}$ was $84 \%$; it was signifi-

Figure 3. Receiver operating characteristic curves of the 4 basic measures. The $\mathrm{CPD}_{\mathrm{fs}}$ data did the best job of discriminating cancerous from noncancerous regions; $\mathrm{CPD}_{\mathrm{pm}}$ and $\mathrm{V}$ had little value as discriminators; NMPCP actually showed an inverse relationship to cancer. The 3 gray regions represent the $95 \%$ confidence limits of the $C P D_{f s}$ curve, the $C P D_{p m}$ and $V$ curves, and the NMPCP curve, respectively. It can be seen that the differences among the 3 sets of curves are significant.

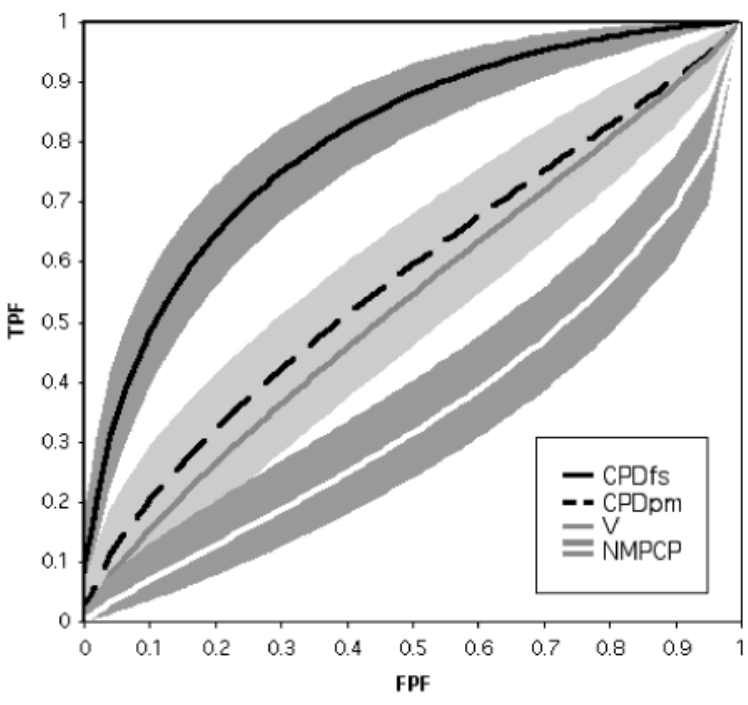


cantly different from $\mathrm{CPD}_{\mathrm{fs}}$ at $P=.07$. Thus, adding the information in NMPCP to $\mathrm{CPD}_{\mathrm{fs}}$ did significantly improve the results.

Next, analysis of the microvessel density data was performed. As described above, the counts were made within the microscope field that visually contained the most endothelial stain within each $0.5-\mathrm{cm}$ square that was counted. This produced a wide distribution of counts within each cancerous and noncancerous region counted. The most distinctive characteristic of cancerous microvessel counts compared with noncancerous regions was in the counts of more than 80 (Fig. 5).

This result implied that the best way to use the microvessel density counts made in this study was to characterize each cancerous region by its highest counts. Next, the correlation of the microvessel density information with the ultrasonographic measures was analyzed. This was done by dividing each prostate's cancerous regions into large $(>0.5$ $\left.\mathrm{cm}^{3}\right)$ and small $\left(<0.5 \mathrm{~cm}^{3}\right)$ and computing the mean ultrasonographic measures and 90thpercentile count for each prostate's divisions. The results show that the ultrasonographic measures were negatively correlated with the high microvessel counts in the large cancers. A correlation of -0.34 was obtained for speed-

Figure 4. Receiver operating characteristic curve of $C P D_{f s}$ combined with NMPCP. The $\mathrm{CPD}_{\mathrm{fs}}$ data had accuracy (area under the curve) of $80.46 \%$, and the reciprocal of NMPCP had accuracy of $64.75 \%$. The best linear combination of these measures was $C P D_{f s}-3.3$ NMPCP, which had accuracy of $83.36 \%$. The 1 -sided $P$ value that this combined measure's accuracy was better than that of $C \mathrm{CD}_{\mathrm{fs}}$ was .07. This indicates that the information in the power mode values does contribute to the discrimination of prostate cancer.

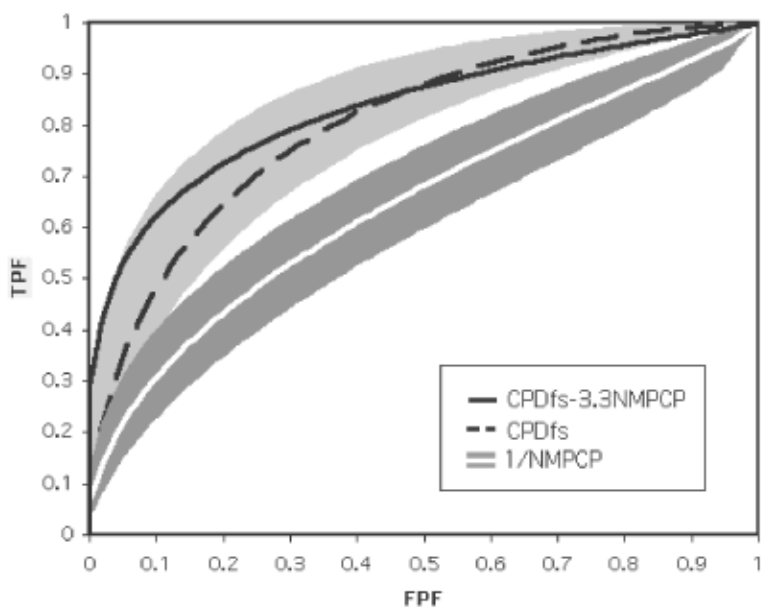

weighted pixel density in large cancers, and a correlation of -0.19 was obtained in small cancers.

Mixed results were obtained when microvessel density was investigated previously as a means to determine the aggressiveness of cancer by comparing it with surgical stage. ${ }^{6,14-19}$ In Table 1, our results show that for this study a high microvessel density was more likely to occur in prostates that had organ-confined cancer.

\section{Discussion}

The purpose of this study was to determine whether quantitative ultrasonographic color flow measures provide information that could be used to identify prostate cancer. It is important to remember, however, that this study was not designed to determine the clinical efficacy of Doppler ultrasonography; therefore, the results are intended to determine which of the studied Doppler ultrasonographic measures is best. Determining the effectiveness of the best Doppler ultrasonographic measure for identifying prostate cancer will be left to a future study designed for that purpose.

That said, of the 4 basic quantitative ultrasonographic measures, $\mathrm{CPD}_{\mathrm{fs}}$ provided the best discrimination of cancer in the prostate. It is not readily apparent why $\mathrm{CPD}_{\mathrm{fs}}$ did so much better than $\mathrm{CPD}_{\mathrm{pm}}$ at discriminating cancer. It cannot be explained by the relative sensitivity of the 2 modalities; globally, over all the prostates, $16 \%$ of the voxels contained power

Figure 5. Comparison of microvessel density distribution in cancerous and noncancerous regions. The distribution of counts within cancerous regions from all prostates is shown to differ significantly from the distribution range of noncancerous counts (gray area) only at the highest counts (>80). Counts made in regions adjacent to cancer did not deviate as much from the distribution range of noncancerous counts.

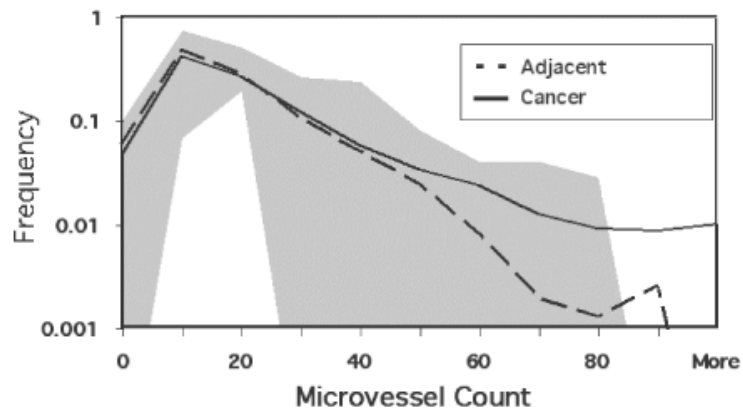


Table 1. Occurrence of High Microvessel Density With Respect to Stage and Grade

\begin{tabular}{|c|c|c|c|c|c|c|c|c|c|c|}
\hline \multirow[b]{2}{*}{ Extent of Cancer } & \multicolumn{2}{|c|}{ Noncancerous } & \multicolumn{2}{|c|}{ Adjacent } & \multicolumn{2}{|c|}{ Gleason 4 and 5} & \multicolumn{2}{|c|}{ Gleason 6} & \multicolumn{2}{|c|}{ Gleason 7-9 } \\
\hline & $n$ & $\%$ & $\mathrm{n}$ & $\%$ & $n$ & $\%$ & $\mathrm{n}$ & $\%$ & $n$ & $\%$ \\
\hline Adverse disease & 16 & 12.5 & 16 & 0.0 & 5 & 0.0 & 11 & 0.0 & 15 & 20.0 \\
\hline Organ confined & 23 & 0.0 & 23 & 13.0 & 8 & 0.0 & 22 & 31.8 & 11 & 36.3 \\
\hline
\end{tabular}

Shown are the percentages of prostates in this study with microvessel density counts of more than 80 in cancers of the given Gleason grade range. Adjacent indicates microvessel counts made within the $0.5-\mathrm{cm}$ squares adjacent to cancerous regions; $n$, total number of prostates that had regions of that category; and \%, percentage of prostates that had counts of more than 80 within that category. The prostates were classified by surgical stage. It can be seen that the microvessel density is most likely to be high in organ-confined cancers of Gleason grade 7 and higher.

mode color, whereas only $10 \%$ had frequency shift color. Power mode information is more depth dependent than frequency shift information; however, this information was probably not a factor in the CPD measures, which were bimodal in nature.

With the assumption that a majority of the color pixels that exist in the frequency shift data are common with the power mode, which is supported qualitatively by visual analysis of the data, the only remaining explanation is that the additional $6 \%$ of color pixels seen in the power mode have a confounding effect. Further analysis aimed at identifying those pixels may provide further insights and may help improve the utility of the power mode for prostate cancer detection. The mean value in the power mode (NMPCP) had an ROC curve with an area of less than $50 \%$ (35\%), which means that low power values are more indicative of cancer. This result is not entirely surprising, because it suggests that the smaller vessels, which have a lower power mode signal, will be found in greater concentrations in cancerous tissue. When the information contained in NMPCP is added to that of $\mathrm{CPD}_{\mathrm{fs}}$ in a Bayesian optimized linear combination, there is an improvement, as shown in Figure 4. This improvement is significant at $P=0.07$ that the 2 areas are different. This result suggests that the use of contrast agents, which would increase the sensitivity of ultrasonography for visualizing small vessels, could improve its ability to detect prostate cancer.

It is still an area of debate whether microvessel density is useful for classifying cancer. Typically, studies of microvessel density investigate how well it correlates with other prognostic indicators such as the extent of cancer spread (organ confined versus various degrees of adverse disease) and Gleason grade. Microvessel density is not used to differentiate cancer from noncancerous tissue because other histologic methods exist for that.

In this study, we wished to use microvessel density to gain some understanding about the distribution of blood vessels in and around cancers and how that relates to what is seen on ultrasonography. First, the overall distribution of microvessel density was looked at in both noncancerous and cancerous tissue of different classifications. It can be seen that the cancerous distributions differ significantly from the noncancerous distributions only at the high end ( $>80$ microvessel counts per field). This means that the most important information contained in the microvessel density is found in the highest counts within a given region, which is consistent with the methods of other studies that used the area of highest endothelial staining to characterize a whole cancerous region. ${ }^{14,15,18}$

When we looked at the fraction of different classifications of cancer with counts of more than 80 , we saw that there was a correlation with Gleason grade. However, organ-confined tumors seemed to be more likely to have a high count than the more dangerous adverse-disease cancers.

Next, we looked at the correlation between microvessel count (90th-percentile count within a given cancer) and selected ultrasonographic measures. In small cancers, the correlation was almost 0 , probably because of the poor signalto-noise ratio in both types of measures. In large cancers, a small negative correlation was seen. Both ultrasonographic and microvessel measures were on average greater in cancer than in noncancerous tissue, so this negative correlation implies that the cancers with high microvessel density tend to have lower ultrasonographic measures and vice versa.

The correlation result was computed to test that hypothesis. A positive correlation result would 
have supported that hypothesis with the argument that regions of high microvessel density correlate with an overall increase in microvascularity, which in turn correlates with a greater number of large ultrasonographically visible vessels.

The negative correlation result that was obtained indicates that we did not prove the hypothesis that there would be a positive correlation between cancers with high ultrasonographically visible blood flow and high microvessel density. However, it is difficult to assign any further meaning to this result without further analysis. For instance, one possible explanation is that regions with high peak microvessel density correlate with regions of low average microvessel density. This explanation is counterintuitive and would have to be tested directly by computing the average microvessel density. A more plausible explanation might be that areas of highest microvascularity do not occur near large vessels.

In summary, the 2 simple components of Doppler ultrasonography that contribute to discriminating prostate cancer from undiseased prostate are $\mathrm{CPD}_{\mathrm{fs}}$ and negative NMPCP. The failure to show a positive correlation between microvessel counts and the ultrasonographic measures indicates that if there is a relationship between the blood flow measured by ultrasonography and the tiny vessels seen histologically, it is a complex relationship that would require a more intricate series of studies to understand.

\section{References}

1. Landis SH, Murray T, Bolden S, Wingo PA. Cancer statistics, 1999 [see comments]. CA Cancer J Clin 1999; 49:8-31.

2. Coley CM, Barry MJ, Fleming C, Wasson JH, Fahs MC, Oesterling JE. Should Medicare provide reimbursement for prostate-specific antigen testing for early detection of prostate cancer? Part II: early detection strategies. Urology 1995; 46:125-141.

3. Ellis WJ, Brawer MK. The significance of isoechoic prostatic carcinoma. J Urol 1994; 152:2304-2307.

4. Vashi AR, Wojno KJ, Gillespie B, Oesterling JE. A model for the number of cores per prostate biopsy based on patient age and prostate gland volume. J Urol 1998; 159:920-924.
5. Newman JS, Bree RL, Rubin JM. Prostate cancer: diagnosis with color Doppler sonography with histologic correlation of each biopsy site [see comments]. Radiology 1995; 195:86-90.

6. Patel U, Rickards D. The diagnostic value of colour Doppler flow in the peripheral zone of the prostate, with histological correlation. $\mathrm{Br} J$ Urol 1994; 74:590-595.

7. Ismail M, Petersen RO, Alexander AA, Newschaffer C, Gomella LG. Color Doppler imaging in predicting the biologic behavior of prostate cancer: correlation with disease-free survival. Urology 1997; 50: 906-912.

8. Okihara K, Kojima M, Nakanouchi T, Okada K, Miki $\mathrm{T}$. The role of power Doppler-guided prostatic biopsy in the diagnosis of prostate cancer. Hinyokika Kiyo 1999; 45:559-563.

9. Moskalik AP, Carson PL, Fowlkes JB, Rubin JM, Bree R. An experimental model for histopathologic correlation of color Doppler of the prostate. Radiol Soc North Am Electronic J 1999. Available at: http://ej.rsna.org/ej3/0102-99.fin/index.html.

10. Moskalik A, Meyer C, Carson P, Rubin J, Fowlkes J, Bree R. 3D registration of ultrasound with histology in the prostate for comparison of quantitative color flow measures with tissue type and microvessel density. In: 1997 Symposium Proceedings. Piscataway, NJ: Institute for Electrical and Electronics Engineers; 1997:1397-1400.

11. Rubin JM, Bude RO, Fowlkes JB, Spratt RS, Carson $\mathrm{PL}$, Adler RS. Normalizing fractional moving blood volume estimates with power Doppler US: defining a stable intravascular point with the cumulative power distribution function. Radiology 1997; 205: 757-765.

12. Swetts JA, Pickett RM. Fundamentals of accuracy analysis. In: Evaluation of Diagnostic Systems: Methods From Signal Detection Theory. New York, NY: Academic Press; 1982:15-45.

13. Obuchowski NA, Lieber ML, Powell KA. Data analysis for detection and localization of multiple abnormalities with application to mammography. Acad Radiol 2000; 7:516-525.

14. Weidner N, Carroll PR, Flax J, Blumenfeld W, Folkman J. Tumor angiogenesis correlates with metastasis in invasive prostate carcinoma. Am J Pathol 1993; 143:401-409. 
15. Rubin MA, Buyyounouski $M$, Bagiella $E$, et al. Microvessel density in prostate cancer: lack of correlation with tumor grade, pathologic stage, and clinical outcome. Urology 1999; 53:542-547.

16. Gettman MT, Pacelli A, Slezak J, et al. Role of microvessel density in predicting recurrence in pathologic stage T3 prostatic adenocarcinoma. Urology 1999; 54:479-485.

17. Gettman MT, Bergstralh EJ, Blute $M$, Zincke $H$, Bostwick DG. Prediction of patient outcome in pathologic stage T2 adenocarcinoma of the prostate: lack of significance for microvessel density analysis. Urology 1998; 51:79-85.

18. Brawer MK. Quantitative microvessel density. A staging and prognostic marker for human prostatic carcinoma. Cancer 1996; 78:345-349.

19. Fregene TA, Khanuja PS, Noto AC, et al. Tumorassociated angiogenesis in prostate cancer. Anticancer Res 1993; 13:2377-2381. 